\title{
Cloud Free Line of Sight Prediction for Low Earth Orbit Optical Satellite Networks
}

\section{Nikolaos K, Lyras, \\ Charilaos I. Kourogiorgas, Athanasios D. Panagopoulos}

Radio \& Satellite Communications Group School of Electrical and Computer Engineering, National Technical University of Athens, Greece

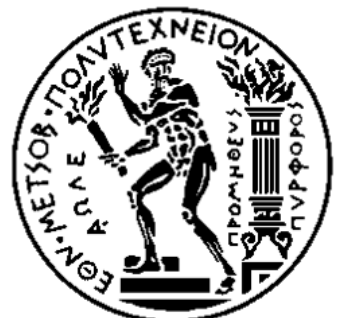




\section{Outline}

- Introduction

- System Consideration

- Methodology

- Numerical Results

- Conclusion/Future Work 


\section{Introduction}

- Why Optical Communications?

- High Data Rates, More Spectral Bandwidth

- Narrow Beams: No Interception, Improved Security, No Regulation

- Low size, mass and power consumption

- Near Earth

- GEO

- NGSO

- Deep Space 


\section{Introduction/Impairments}

- Propagation Impairments

- Liquid Water Clouds (Blockage)

- Cirrus Clouds

- Absorption/Scattering due to atmospheric gasses aerosols

- Diffraction due to free space propagation

- Turbulence 


\section{Cloud Coverage}

\section{Cloud Coverage}




\section{Cloud Coverage}

- Liquid Water Clouds

- Dominant fading mechanism

- Blockage of the link

- Mitigation Technique

- Site Diversity

- Optical Ground Station Network (OGSN)

- Large Distances

- Uncorrelated paths 


\section{CFLOS modeling}

- Methodologies for the Computation of Cloud Free Line of Sight (CFLOS) Probability

- Proposed CFLOS modeling

- Single and Joint CFLOS Statistics

- Spatial and Temporal variability

- Vertical Extent of Clouds

- Elevation Angle

- Altitude for high altitude stations 


\section{System Considerations}




\section{System Considerations}

- OGS-LEO Satellite

- Varying Elevation Angles with Time

- Elevation Angles>20deg

- CFLOS Estimation

- Single Link: LEO satellite is within the visibility area of the OGS

- OGSN/Joint CFLOS: At least 1 OGS is within the visibility area of the LEO sat.

- Sub-Satellite Points: AGI/STK tool

- Iridium LEO satellite

- Continental Diversity Scenario 


\section{Systems Consideration}

- PDF of elevation angle

- OGS located in Heraklion, Crete

- Iridium LEO satellite (altitude: $779 \mathrm{~km}$, inclination angle $88.9 \mathrm{deg}$ )

- Sub-satellite for 1 year-30sec temporal resolution.

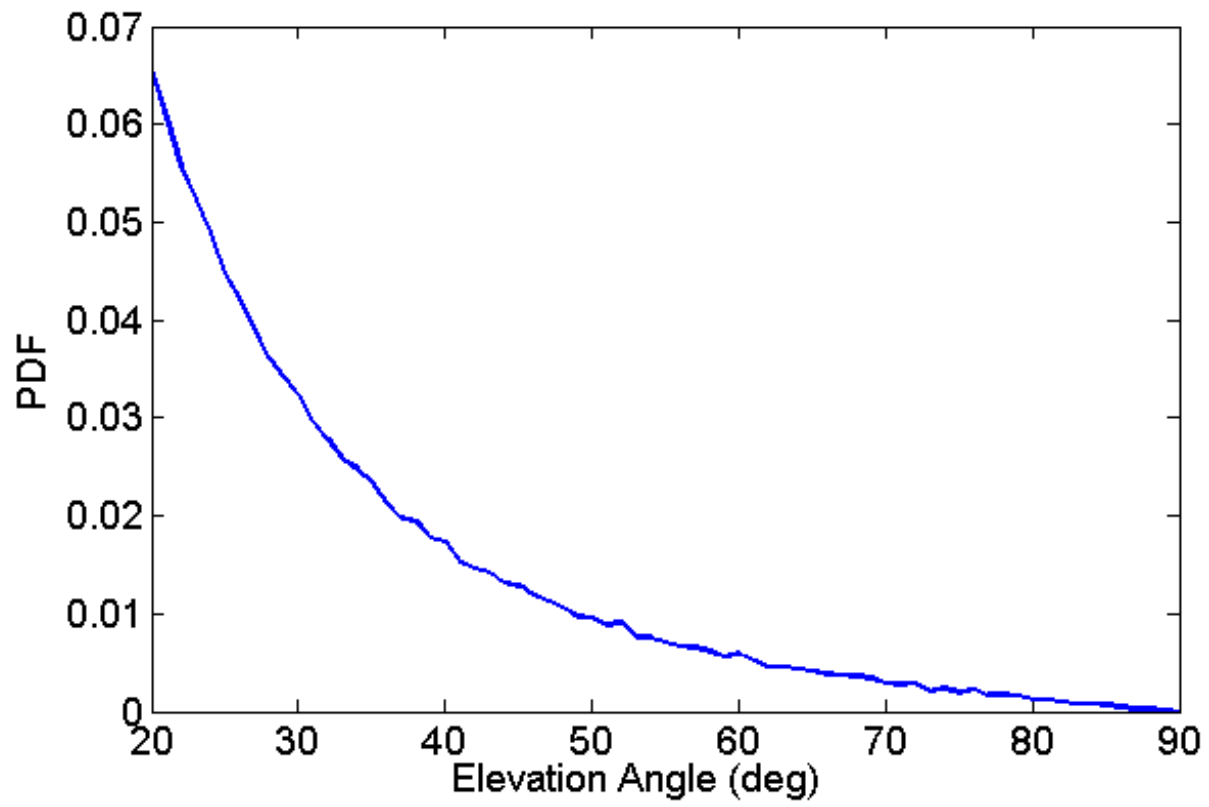




\section{Stochastic CFLOS Time Series Generator}




\section{CFLOS modeling/ Time series Generator}

- Liquid Water Clouds

- Liquid Water Content (LWC)

- Depending on Height

- Integrated Liquid Water Content (ILWC)

- Described by Lognormal Distribution

- CFLOS time series synthesizer

- On/Off channel

- ILWC>0 $\Rightarrow$ CLOUD

- ILWC statistical parameters derived from meteorological databases 


\section{Methodology}

- ILWC 2-D map for a specific time instance for a specific place

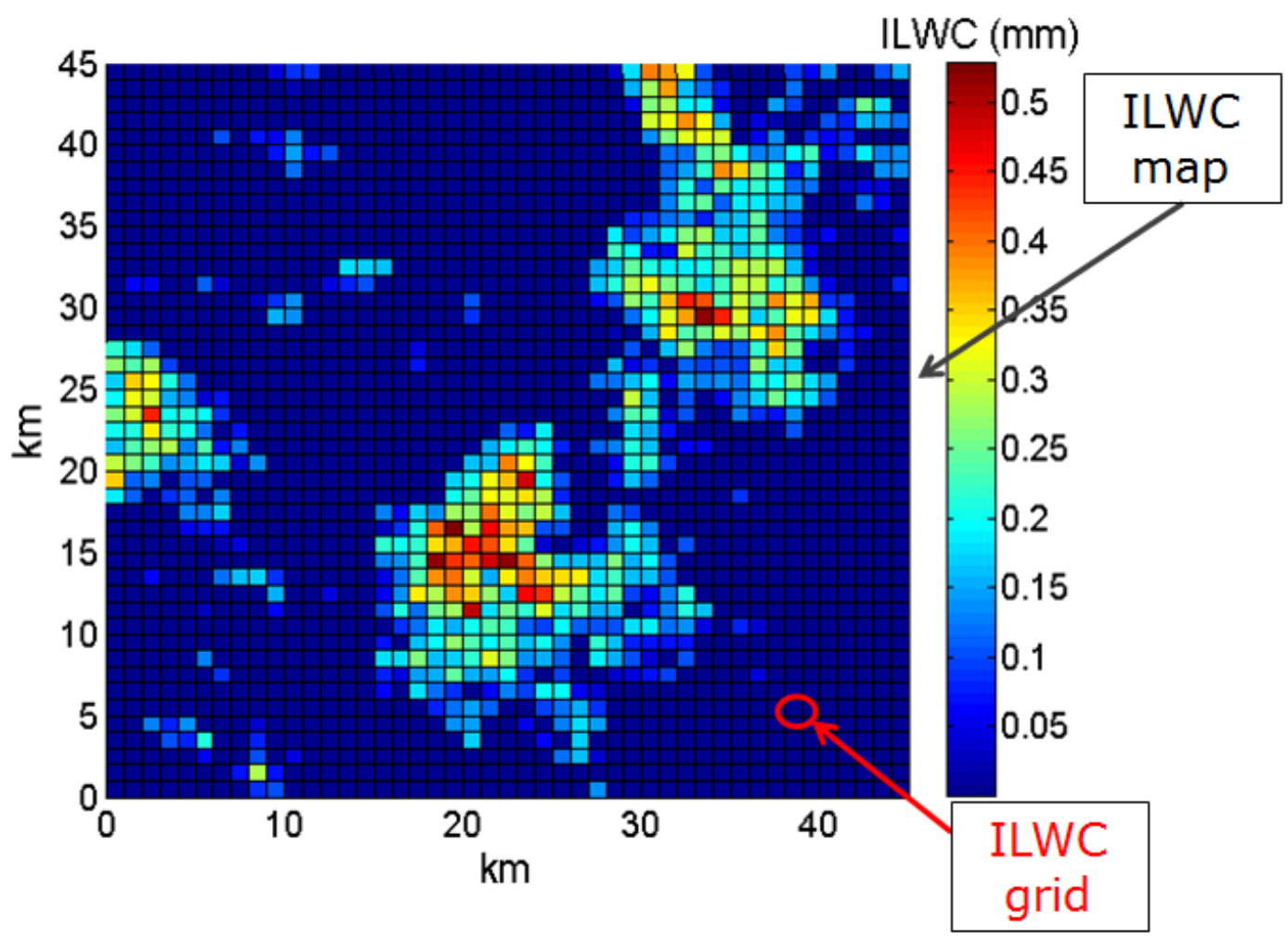

Lyras, N.K., Kourogiorgas, C.I. and Panagopoulos, A.D., "Cloud Attenuation Statistics Prediction From Ka-Band to Optical Frequencies: Integrated Liquid Water Content Field Synthesizer," in IEEE Transactions on Antennas and Propagation, vol. 65, no. 1, pp. 319-328, Jan. 2017. 


\section{ILWC time Series Generator}

- Stochastic modeling of ILWC (L)

- For each place of interest (OGS) an ILWC map is synthesized

- Each ILWC map consists of grids/fields of ILWC correlated on Temporal and on Spatial domain

$$
\boldsymbol{L}_{\text {map }, \text { oGs }}=\left[\boldsymbol{L}_{1}, \boldsymbol{L}_{2}, \ldots, \boldsymbol{L}_{n}\right]
$$

- For $k$ OGS, an ILWC map for each OGS is generated

$$
L_{\text {total_field }}=\left[\begin{array}{llll}
L_{\text {map }, O G S^{1}} & L_{\text {map }, O G S^{2}} & \ldots & L_{\text {map }, O G S^{k}}
\end{array}\right]
$$

- ILWC maps/grids are correlated one to each other on temporal and spatial domain 


\section{ILWC time Series Generator}

- Stochastic modeling of ILWC (L)

- Each $L$ is given according to:

$$
L_{i}(t)=\left\{\begin{array}{ll}
\exp \left[Q^{-1}\left(\frac{1}{P_{C L W}} Q\left(G_{i}(t)\right)\right) \times \sigma+m\right] & G_{i}(t) \geq \alpha_{t h} \\
0 & G_{i}(t) \leq \alpha_{t h}
\end{array}\right\}
$$

- $m, \sigma, P c / w$ are the statistical parameters of ILWC for each place of interest, derived from data bases (e.g. ERA Interim)

- $G_{i}(t)$ is the Gaussian process for the generation of each grid. Stochastic Differential Equations are used. Spatial and temporal correlation is taken into account.

- All elements of $L_{\text {total_field }}$ are temporally and spatially correlated 


\section{ILWC time Series Generator}

- Stochastic modeling of ILWC (L)

- Generation of $G_{i}(t)$ :

$$
G_{i}(t)=\gamma_{1} \cdot \mathrm{X}_{i}^{1}(t)+\gamma_{2} \cdot \mathrm{X}_{i}^{2}(t),(i=1, \ldots ., n)
$$

- Superposition of two Gaussian processes

- SDE's for each Gaussian process

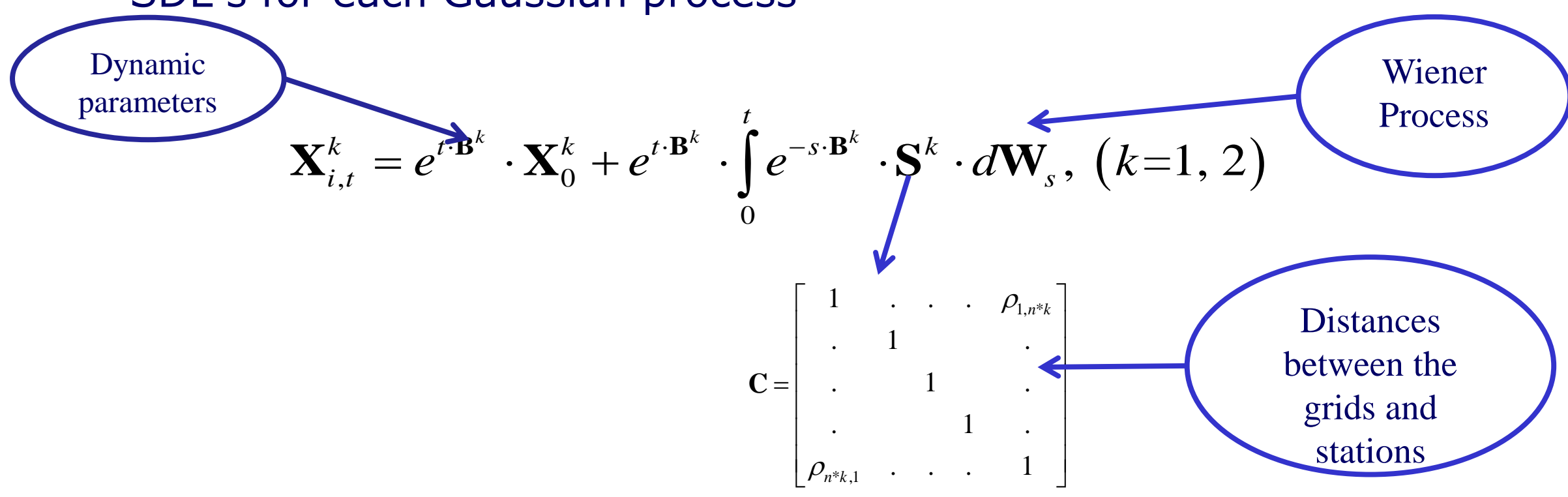




\section{ILWC Time Series Generator}

- ILWC 2-D maps for a for a specific place

- Left time $t=t_{0}$, Right 60 min later
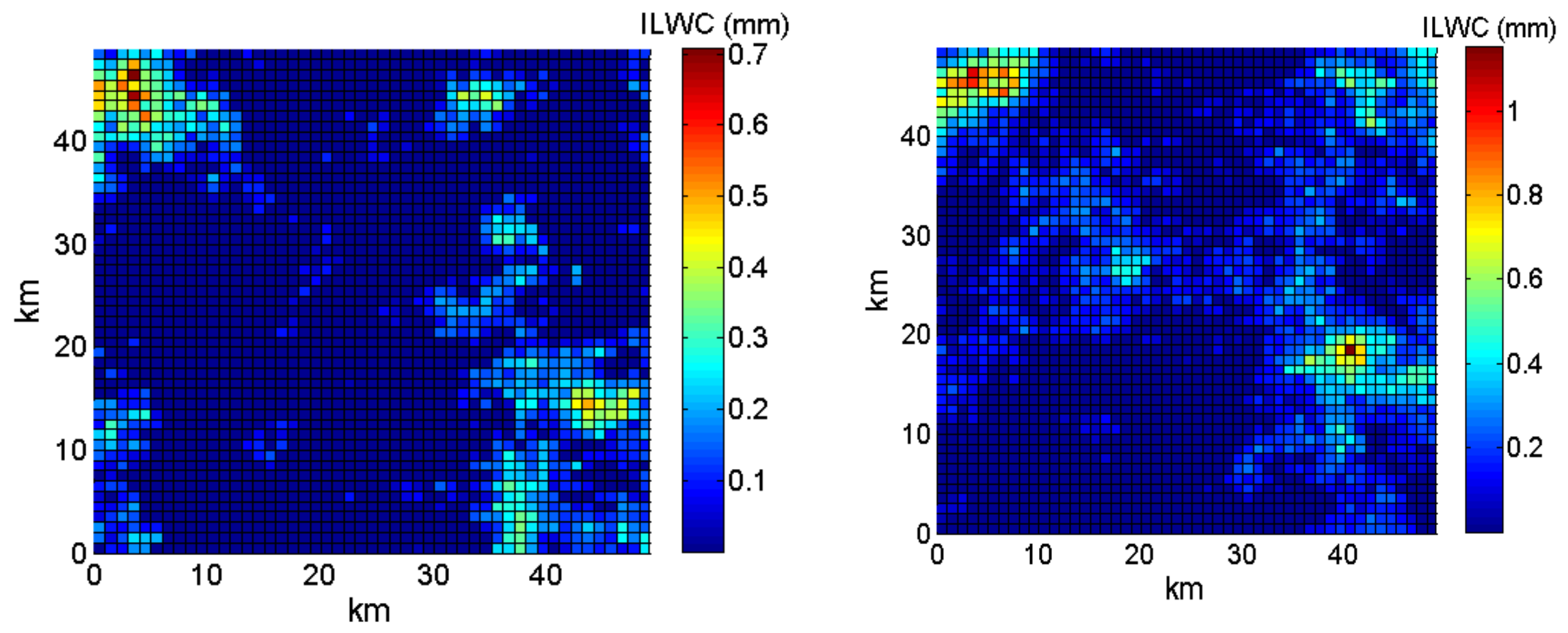

International Conference on Space Optics, ICSO 2018, 9-12 October 2018, Chania, Greece 


\section{Clouds Vertical Extent}

- Why?

- Elevation Angle

- High Altitude Stations

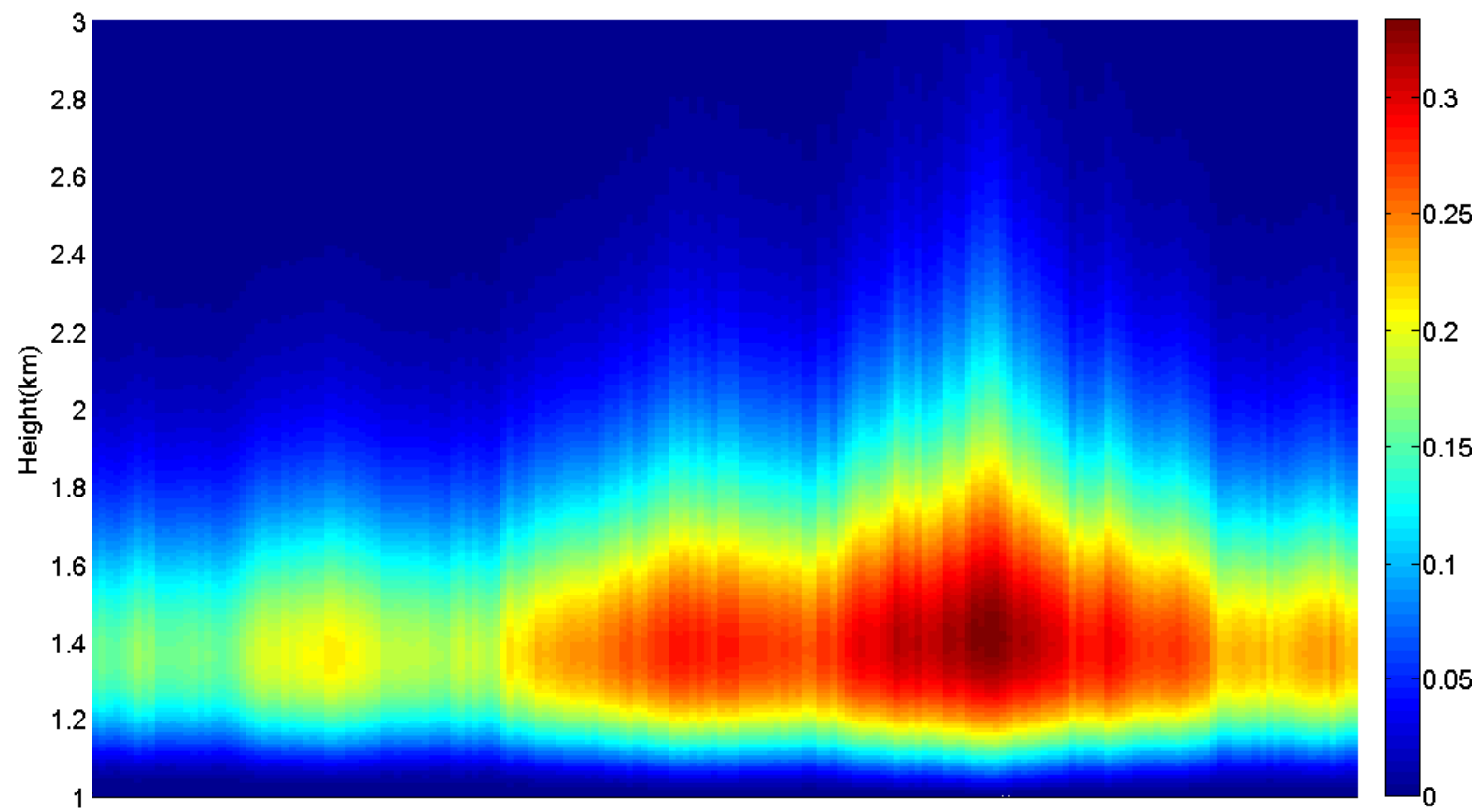

Time(s) 


\section{CFLOS Time Series Generator}

- Clouds Vertical Extent Computation

- For each $L_{i}(t)$ the vertical extent of the cloud grid is computed

$\tilde{w}_{i}(h, t)= \begin{cases}\frac{L_{i}(t)}{c_{i}^{2}(t)^{c_{i}^{1}(t)} \Gamma\left(c_{i}^{1}(t)\right)}\left(h-h_{0}\right)^{c_{i}^{1}(t)-1} e^{-\left(h-h_{0}\right) / c_{i}^{2}(t)} & \text { for } h \geq h_{0} \\ 0 & \text { for } h<h_{0}\end{cases}$

- $\tilde{w}(h, t) \rightarrow 0 \quad$ for $\quad \tilde{w}_{i}(h, t) \leq 0.06 \cdot L_{i}(t)$

- Vertical extent known for each

Grid in ILWC map

- Single joint CFLOS statistics

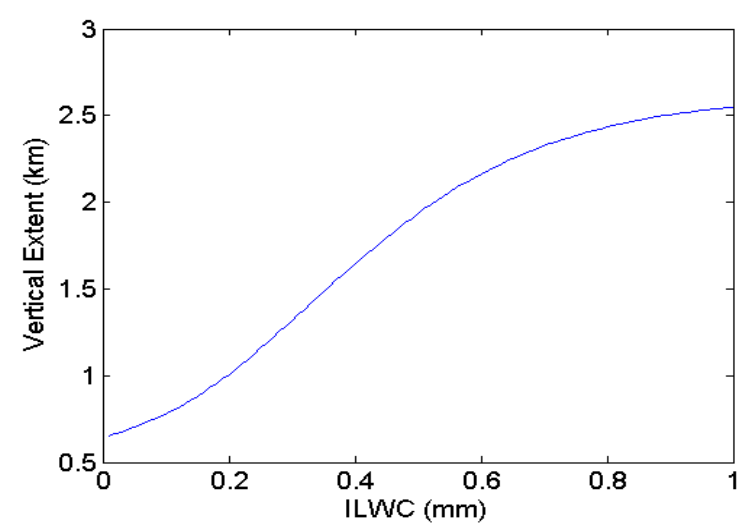




\section{CFLOS Time Series Generator}

- ILWC 3-D maps:

- left time $t=t_{o}$, Right 60 min later
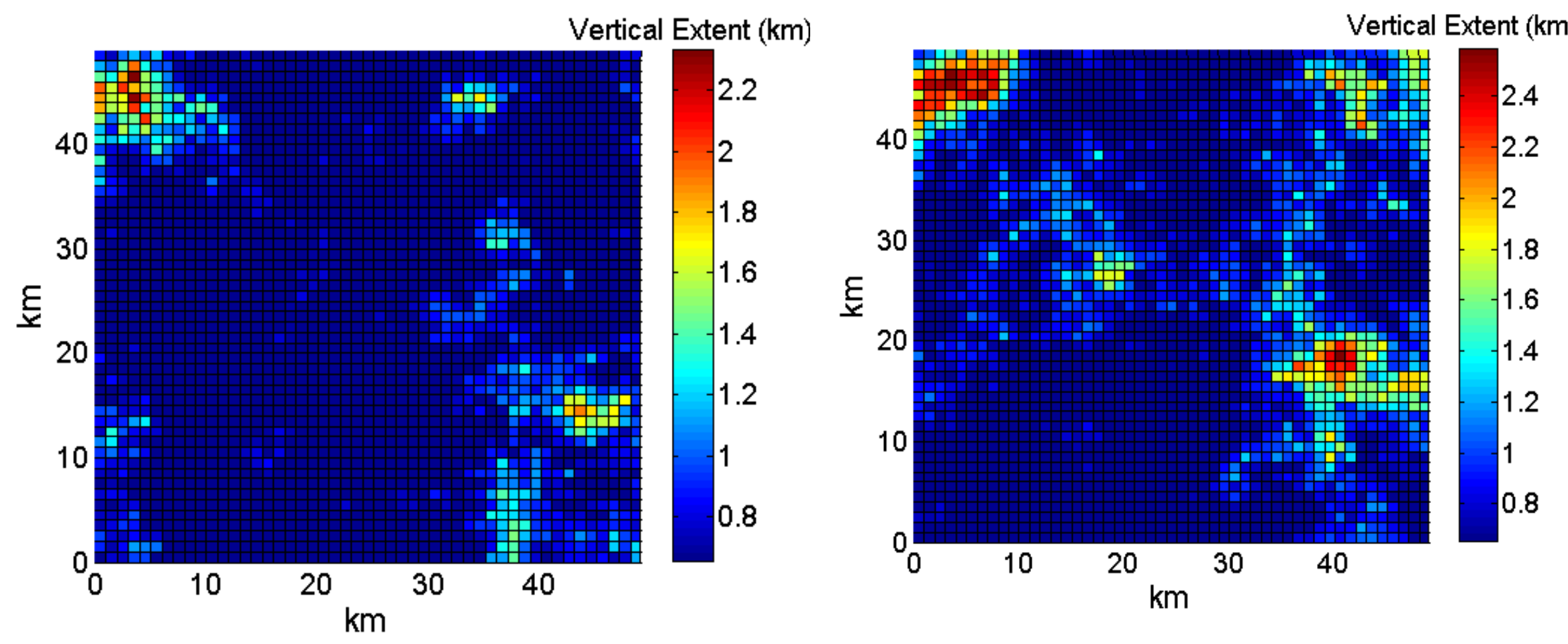

International Conference on Space Optics, ICSO 2018, 9-12 October 2018, Chania, Greece 


\section{CFLOS Time Series Generator}

- OGS CFLOS estimation

- For each station: if there is at least one cloud grid (grid with ILWC $>0$ ) with vertical extent that impairs the link along the slant path then the whole optical slant path is considered blocked

- Elevation angle is taken into account

- For OGSN: if at least one station is not blocked by clouds, then the OGSN is considered free of clouds and the joint CFLOS statistics can be calculated 


\section{CFLOS Time Series Generator}

- Synthesizer Main Steps

- AGI/STK tool the sub satellite points of LEO Satellite are computed

- Elevation angles for each OGS using the sub satellite points are computed

- ILWC maps (time series) for each OGS are generated using the proposed methodology

- Vertical extent for each ILWC grid is computed. 2-D ILWC maps are converted to 3D maps

- For each elevation angle CFLOS time series are computed 


\section{Numerical Results}




\section{Numerical Results}

- ILWC first order statistics validation

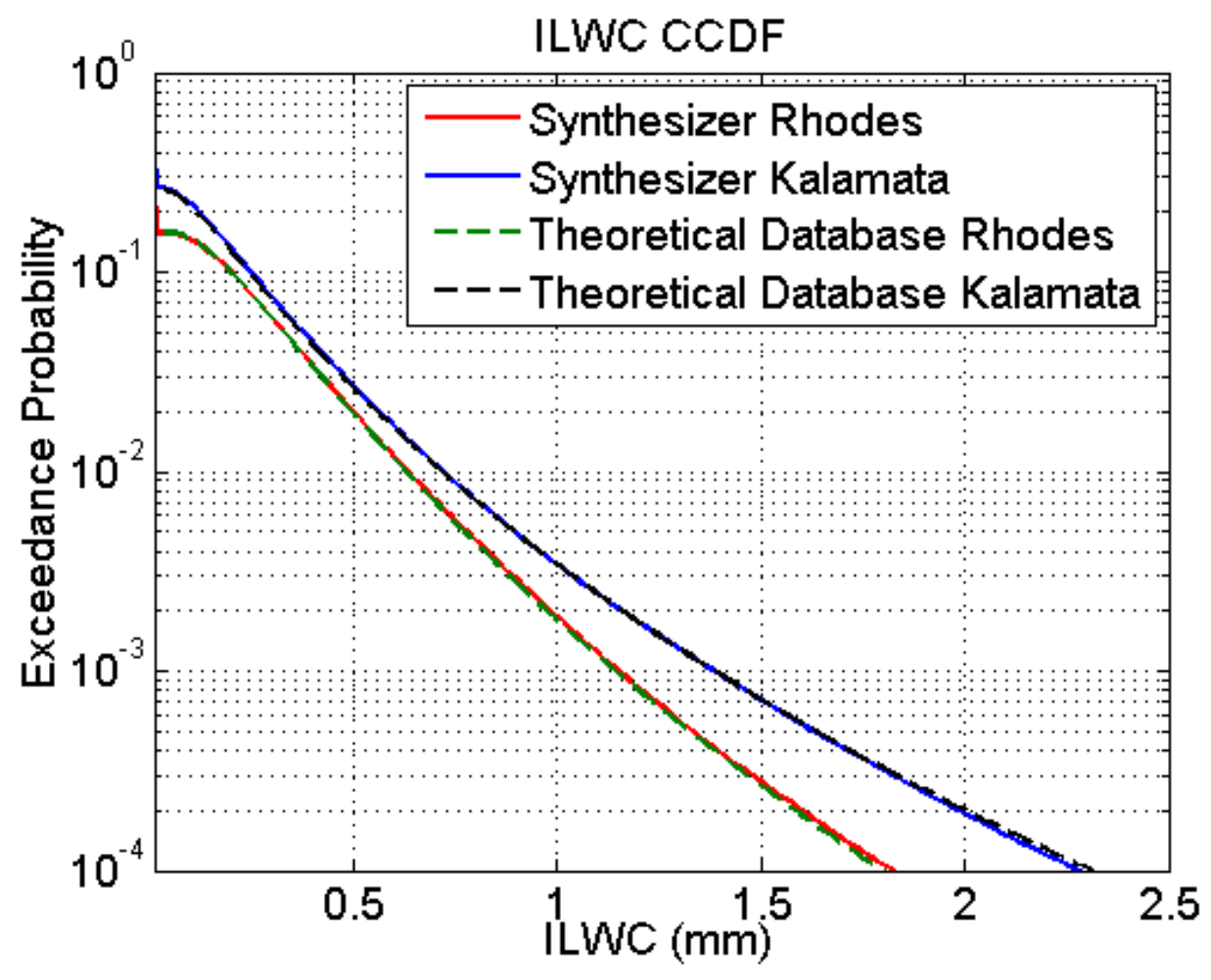

- ILWC space-time synthesizer reproduces the firstorder (exceedance probability) statistics of ILWC 


\section{Numerical Results}

- CFLOS for different elevation angles

- Statistical parameters of ILWC are derived from ITU-R databases (ECMWF ERA-40)

- Hypothetical link in Naxos, Greece

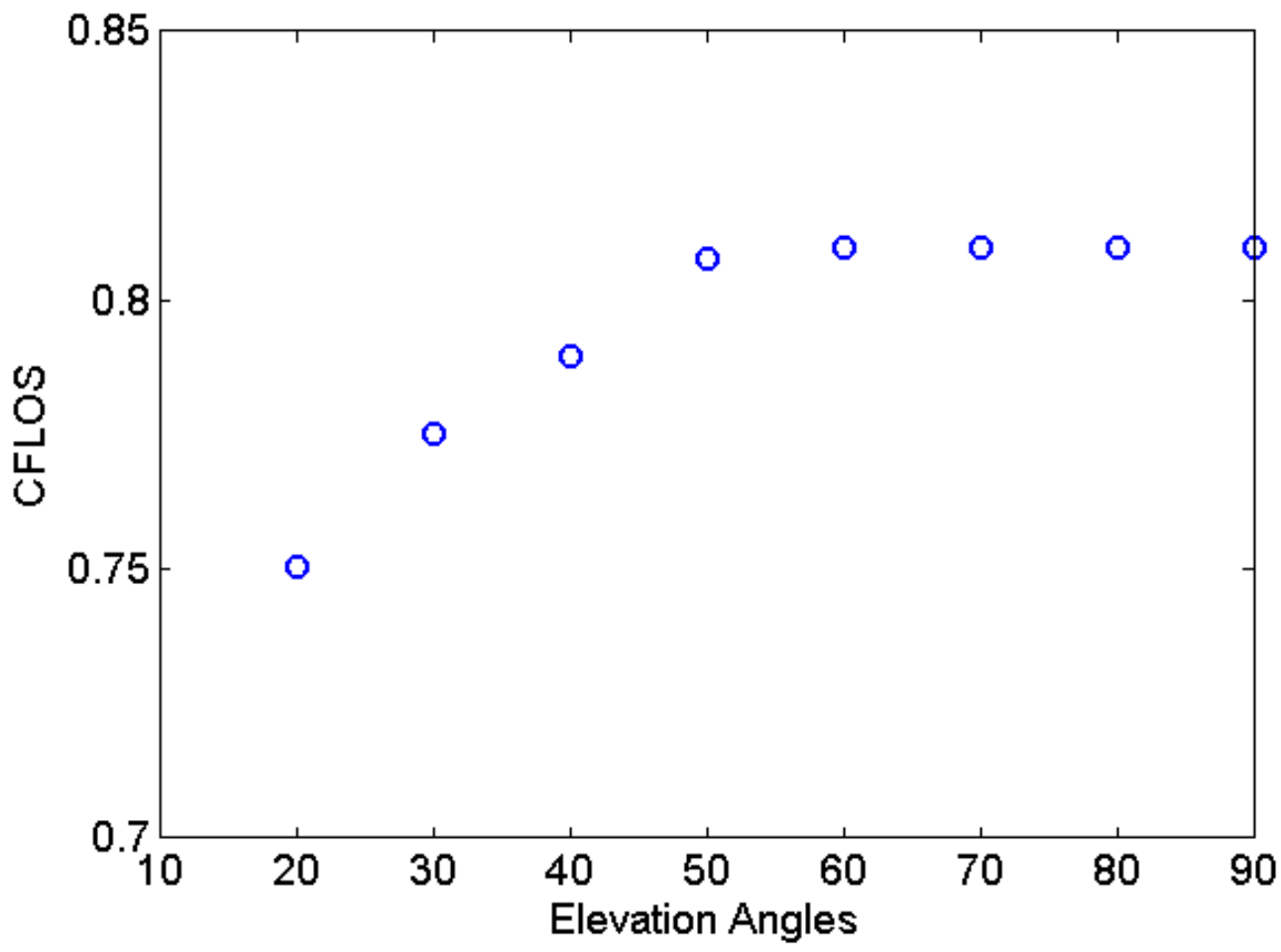




\section{Numerical Results}

- Single/Joint CFLOS, Iridium LEO satellite

- ERA Interim database (ECMWF) (2000 -2016)

- 30sec temporal resolution

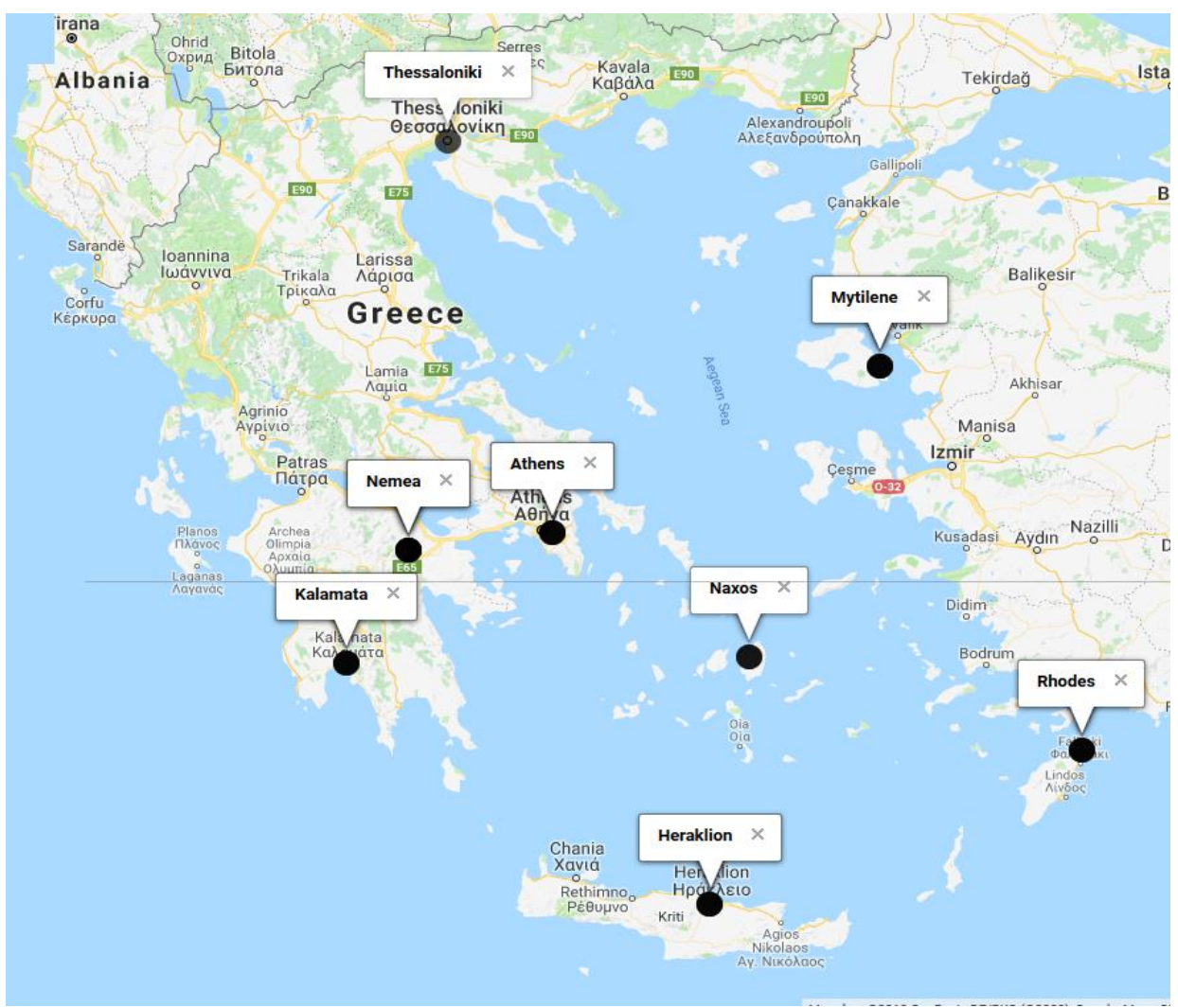




\section{Numerical Results}

- Estimation of Single CFLOS

\begin{tabular}{|c|c|c|c|c|}
\hline Location & $\begin{array}{c}\text { Latitude } \\
(\mathbf{d e g})\end{array}$ & $\begin{array}{c}\text { Longitude } \\
\mathbf{( d e g )}\end{array}$ & $\begin{array}{c}\text { Min-Max } \\
\text { Elev. }(\mathbf{d e g})\end{array}$ & $\begin{array}{c}\text { CFLOS } \\
(\%)\end{array}$ \\
\hline Nemea & 37.84 & 22.62 & $20-89.4$ & 55.6 \\
\hline Heraklion & 35.33 & 25.13 & $20-89.86$ & 69.1 \\
\hline Athens & 37.96 & 23.82 & $20-89.6$ & 62.2 \\
\hline Kalamata & 37.05 & 22.1 & $20-89.6$ & 61.4 \\
\hline City of Rhodes & 36.43 & 28.22 & $20-89.4$ & 71.7 \\
\hline Mytilene & 39.11 & 26.54 & $20-89.5$ & 64.6 \\
\hline Thessaloniki & 40.64 & 22.95 & $20-89.8$ & 53.5 \\
\hline Naxos & 37.09 & 25.38 & $20-89.3$ & 68.3 \\
\hline
\end{tabular}

- Joint CFLOS 91.32\% 


\section{Conclusion/Future work}

- Stochastic CFLOS Time Series Generator

- ILWC statistics are used

- 3D ILWC synthesizer

- Spatial and Temporal Variability of ILWC

- Vertical Extent of clouds

- Single and Joint CFLOS statistics

- LEO Satellite-OGSs in Greece

- Varying Elevation angles

- Future Work

- Second Order Statistics Analysis

- Handovers

- Incorporation of LEO constellation

- Dedicated Studies for the height of cloud base and the vertical extent of clouds especially for high altitude stations 


\title{
Thank You for your Attention!
}

\author{
email: lyrasnikos@central.ntua.gr
}

